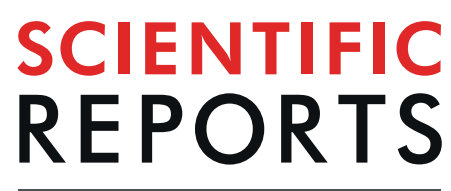

natureresearch

\title{
OPEN The expression of the long NEAT1_2 isoform is associated with human epidermal growth factor receptor 2-positive breast cancers
}

\begin{abstract}
Erik Knutsen ${ }^{1}$, Seyed Mohammad Lellahi ${ }^{1}$, Miriam Ragle Aure ${ }^{2}$, Silje Nord ${ }^{2}$, Silje Fismen ${ }^{3,23}$, Kenneth Bowitz Larsen ${ }^{1}$, Marta Tellez Gabriel ${ }^{1}$, Annica Hedberg ${ }^{1}$, Sunniva Stordal Bjørklund ${ }^{2}$, Oslo Breast Cancer Research Consortium (OSBREAC) ${ }^{4 \dagger}$, Anna Mary Bofin ${ }^{5}$, Gunhild Mari Mælandsmo ${ }^{6}$, Therese Sørlie ${ }^{2}$, Elin Synnøve Mortensen ${ }^{1,3}$ \& Maria Perander ${ }^{1 *}$

The long non-coding RNA NEAT1 locus is transcribed into two overlapping isoforms, NEAT1_1 and NEAT1_2, of which the latter is essential for the assembly of nuclear paraspeckles. NEAT1 is abnormally expressed in a wide variety of human cancers. Emerging evidence suggests that the two isoforms have distinct functions in gene expression regulation, and recently it was shown that NEAT1_2, but not NEAT1_1, expression predicts poor clinical outcome in cancer. Here, we report that NEAT1_2 expression correlates with HER2-positive breast cancers and high-grade disease. We provide evidence that NEAT1_1 and NEAT1_2 have distinct expression pattern among different intrinsic breast cancer subtypes. Finally, we show that NEAT1_2 expression and paraspeckle formation increase upon lactation in humans, confirming what has previously been demonstrated in mice.
\end{abstract}

The long non-coding RNA (lncRNA) NEAT1 (Nuclear Paraspeckle Assembly Transcript 1) has recently gained considerable attention as it is abnormally expressed in human diseases, including cancer and neurodegenerative disorders. The NEAT1 gene is transcribed into two isoforms, NEAT1_1 of $3.7 \mathrm{~kb}$ and NEAT1_2 of $22.3 \mathrm{~kb}$, where NEAT1_1 completely overlaps with the $5^{\prime}$ end of NEAT1_2 $2^{1}$. NEAT1_2 is essential for the assembly of paraspeckles, dynamic nuclear ribonucleoprotein complexes that phase-separate from the nucleoplasm to form liquid drop-like structures ${ }^{2-7}$. In contrast, NEAT1_1 expression is not sufficient to induce paraspeckle formation, and recent reports suggest that NEAT1_1 can localize to structures that are distinct from paraspeckles ${ }^{7,8}$. NEAT1 expression and paraspeckle formation are upregulated in response to a variety of cellular stressors including mitochondrial stress, proteasome inhibition, oncogene-induced replication stress, hypoxia, heat shock, and viral infections ${ }^{9-18}$. It is now generally accepted that NEAT1 and paraspeckles regulate gene expression at both transcriptional and post-transcriptional levels by acting as hubs that sequester specific gene regulatory proteins and mRNAs ${ }^{16-20}$. Several lines of evidence suggest that NEAT1 and paraspeckles play critical roles in stress response pathways in general, and at specific developmental stages. NEAT1 knockout mice display compromised mammary gland development and corpus luteum formation ${ }^{21,22}$. Moreover, it was recently shown that maternal and zygotic NEAT1-depletion frequently led to early developmental arrest at the 16- or 32-cell stage in mouse embryonic cells ${ }^{23}$.

Cancer cells are exposed to a variety of extrinsic and intrinsic stressors like hypoxia, proteotoxicity, DNA damage, and reactive metabolic intermediate ${ }^{24}$. Such malignancy-associated stress has been shown to induce NEAT1 expression and paraspeckle formation in vivo ${ }^{15,16}$. NEAT1 levels are elevated in hypoxic regions of breast cancer cell line xenografts, and skin tumors induced by genotoxic stress in mice, display increased NEAT1

\footnotetext{
${ }^{1}$ Department of Medical Biology, Faculty of Health Sciences, UiT -The Arctic University of Norway, Tromsø, Norway. ${ }^{2}$ Department of Cancer Genetics, Institute for Cancer Research, The Norwegian Radium Hospital, Oslo University Hospital, Oslo, Norway. ${ }^{3}$ Department of Clinical Pathology, University Hospital of North Norway, Tromsø, Norway. ${ }^{4}$ www.osbreac.no, Oslo, Norway. ${ }^{5}$ Department of Clinical and Molecular Medicine, Norwegian University of Science and Technology, Trondheim, Norway. ${ }^{6}$ Department of Tumor Biology, Institute for Cancer Research, The Norwegian Radium Hospital, Oslo University Hospital, Oslo, Norway. ${ }^{23}$ Silje Fismen is deceased. ${ }^{\dagger}$ A comprehensive list of consortium members appears at the end of the paper. *email: maria.perander@uit.no
} 
expression and paraspeckle formation ${ }^{15,16}$. In consistence with these observations, NEAT1 is overexpressed in many cancers $15,16,25,26$. In most cases, NEAT1 expression is associated with aggressive disease and poor clinical outcomes $^{15,16,25,26}$.

Breast cancer is the most common type of cancer in women and covers a broad spectrum of different malignant neoplasms with clinical and genomic heterogeneity ${ }^{27,28}$. In clinical diagnosis, breast cancer is classified according to histological grade, Ki-67 proliferative index, and to the expression of hormone and growth factor receptors estrogen receptor (ER), progesterone receptor (PgR), and human epidermal growth factor receptor 2 (HER2). The classification of breast cancer has been stratified by gene expression profiling leading to the identification of a 50-gene signature (PAM50) that groups breast cancer into luminal A, luminal B, HER2-enriched, basal-like, and normal-like intrinsic subtype ${ }^{29-31}$. Several studies have demonstrated that NEAT1 is required for proliferation and survival of breast cancer cell lines ${ }^{12,16,21,32-35}$. Moreover, NEAT1 is frequently overexpressed in breast tumor samples compared to adjacent normal tissue and is associated with poor overall survival ${ }^{16,34-37}$. Recently, genomic analyses of 360 primary breast tumors showed that the core promoter of the NEAT1 gene is frequently mutated in cancer and most of these mutations are associated with loss of expression in in vitro assay $^{38}$. In addition, focal deletions within the NEAT1 gene were found in $8 \%$ of breast cancers, and mutations are frequently found in the exonic region ${ }^{38,39}$. This suggests that NEAT1 expression might either protect or enhance cancer initiation and progression dependent on tumor stage. Moreover, a growing body of experimental evidence shows that the two NEAT1 isoforms have distinct physiological functions ${ }^{40,41}$. Therefore, it is important to address the relative contribution of NEAT1_1 and NEAT1_2 in cancer progression.

In this study, we have examined the relationship between NEAT1_2 expression and breast cancer subtypes by performing RNA-FISH analyses on core needle biopsies using probes solely recognizing the NEAT1_2 isoform. We report that NEAT1_2 expression associates with HER2-positive breast cancers, and with high tumor grade. This is verified by in silico analyses of microarray data from three independent breast cancer cohorts showing that NEAT1_2 is most highly expressed in luminal B and HER2-enriched cancers. Interestingly, we present evidence suggesting that NEAT1_1 expression shows a distinct distribution among breast cancer subtypes compared to NEAT1_2, being highest in ER-positive luminal A and luminal B cancers. This indicates that the relative expression of NEAT1_1 versus NEAT1_2 varies among the different breast cancer subclasses. Finally, we report that NEAT1_2 and paraspeckle formation are induced in vivo in human luminal epithelial cells during lactation.

\section{Results}

NEAT1_2 expression is associated with high tumor grade and HER2 positive breast cancers. The NEAT1_2 isoform is essential for the assembly of paraspeckles that regulate the expression of specific genes at certain cellular circumstances ${ }^{1,16-20}$. Recently, it was shown that the expression of NEAT1_2, but not NEAT1_1, predicts progression-free survival of ovarian cancer treated with platinum-based chemotherapy ${ }^{15}$. This prompted us to specifically investigate the expression of NEAT1_2 in breast cancer. To determine the relationship between breast cancer subtypes and both NEAT1_2 expression and associated paraspeckle formation, we performed NEAT1_2-specifc RNA-FISH analyses on 74 formalin-fixed paraffin-embedded needle biopsies taken from females at the time of diagnosis of breast cancer. The samples were selected to represent cancers pathologically classified as luminal A $(n=23)$, luminal B $(n=29)$, triple negative/basal-like $(n=14)$ and HER2-positive $(n=8)$. We also included 27 non-cancerous breast samples in the study (23 fibroadenomas, 3 mammary reduction, and 1 BRCA1 prophylactic mastectomy). Cancer cells were identified by experienced pathologists, and NEAT1_2 expression was manually scored from " 0 " to " 3 " based on the presence and morphology of punctuated nuclear signals corresponding to paraspeckles (Fig. 1a). Samples scored as "1", "2", and "3" were defined as NEAT1_2positive. Forty-nine patients (66\%) were positive for NEAT1_2 expression (Fig. 1b). In all cases, the expression was strictly restricted to cancer cells, with no detectable NEAT1_2 signals in surrounding stromal tissue, infiltrating immune cells, or in unaffected breast tissue. In sharp contrast, only 2 out of 27 benign breast tissue samples were NEAT1_2-positive (7.4\%), both samples being scored as "1" (Fig. 1b). Clinicopathological characteristics were acquired from each patient and correlated with NEAT1_2 expression (Table 1). NEAT1_2 levels significantly associated with tumor grade (Chi square test $\mathrm{p}=0.027$; Fig. $1 \mathrm{c}$, Table 1 ), confirming what has previously been reported by others on total NEAT1. Interestingly, NEAT1_2 expression also associated with HER2 positive breast cancers (Chi square test $\mathrm{p}=0.042$; Fig. $1 \mathrm{~d}$, Table 1 ). To verify these results, we analyzed microarray expression data from 381 breast cancer patients (Oslo2) using data generated by a NEAT1_2-specific probe ${ }^{42}$. We confirmed that NEAT1_2 expression was associated with grade (Kruskal-Wallis test $\mathrm{p}=3.169 \mathrm{e}-08$; Fig. $2 \mathrm{a}$ ) and HER2 status (Wilcoxon Rank-Sum test $\mathrm{p}=1.49 \mathrm{e}-06$; Fig. 2b). Intriguingly, we also found that NEAT1_2 expression was significantly lower in ER-positive tumors compared to ER-negative tumors in this cohort (Wilcoxon Rank-Sum test $\mathrm{p}=0.005155$; Supplementary Fig. 1). To further determine the relationship between NEAT1_2 and HER2 expression in the Oslo2 cohort, we investigated the correlation between NEAT1_2 levels and ERBB2 copy number and mRNA expression in HER2-positive and HER2-negative cancers. A close to significant positive correlation was found between NEAT1_2 expression and ERBB2 copy number (Spearman's rank correlation $\mathrm{R}=0.35$, $\mathrm{p}=0.074$; Fig. 2c, left panel), and a significant positive correlation was found between NEAT1_2 and ERBB2 mRNA expression (Spearman's rank correlation $\mathrm{R}=0.56, \mathrm{p}=0.003$; Fig. $2 \mathrm{~d}$, left panel). As expected, no correlation was found between NEAT1_2 and ERBB2 amplification or mRNA expression in HER2-negative cancers (Fig. 2c,d, right panel). Finally, we assessed the expression of NEAT1_2 by RNA-FISH and RT-qPCR in nine breast cancer cell lines classified according to the expression of hormone- and growth factor receptors into ER/ PgR-positive HER2-negative cells (MCF7, T-47D), HER2-positive cells (BT474, HCC1569, SK-BR-3), and triple negative cells (BT549, Hs 578T, MDA-MB-231, MDA-MB-468) ${ }^{43}$. In consistence with previous reports, the morphology, as well as the number and size of NEAT1_2-containing paraspeckles, varied substantially between the different cell lines (Supplementary Fig. 2) ${ }^{44}$. We also observed cell-to-cell variations within each cell line. In general, both the number and size of NEAT1_2-containing punctas were hard to determine as they frequently 


\section{a $\frac{\text { Score 0, Negative }}{\text { No detection of NEAT1_2, or }}$ detection in less than $10 \%$ of tumor cells (tumor sample) or mammary epithelial cells (normal sample). \\ Score 1, Positive \\ Detection of NEAT1_2 in 10-50\% of tumor cells (tumor sample) or mammary epithelial cells (normal sample). \\ Punctuated single structures.}

\section{Score 2, Positive}

Detection of NEAT1_2 in more than $50 \%$ of tumor cells (tumor sample) or mammary epithelial cells (normal sample).

Punctuated single structures.

\section{Score 3, Positive}

Detection of NEAT1_2 in more than

$50 \%$ of tumor cells (tumor sample) or mammary epithelial cells (normal sample).

Overlapping large structures.
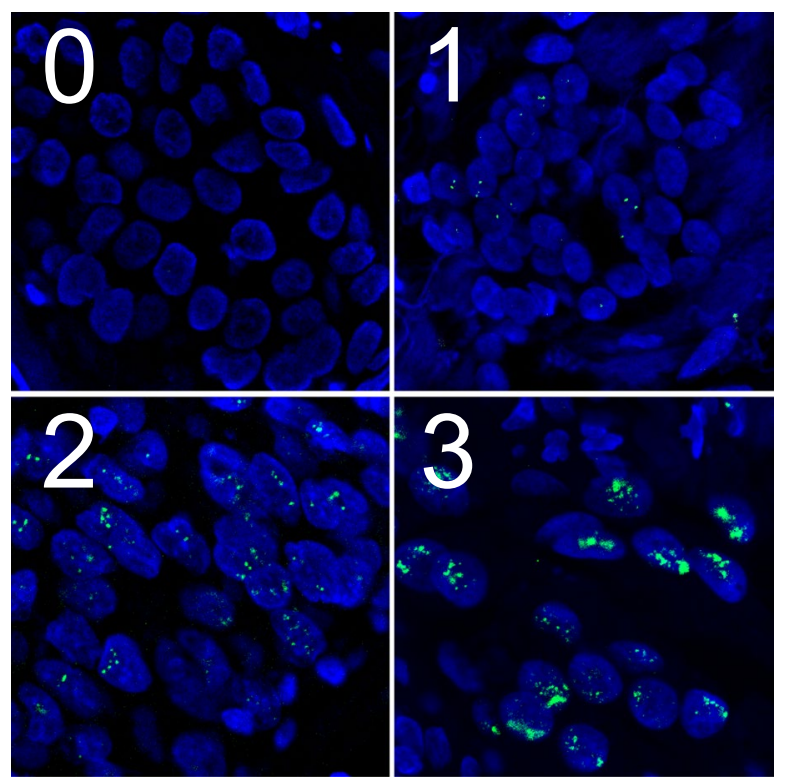

b

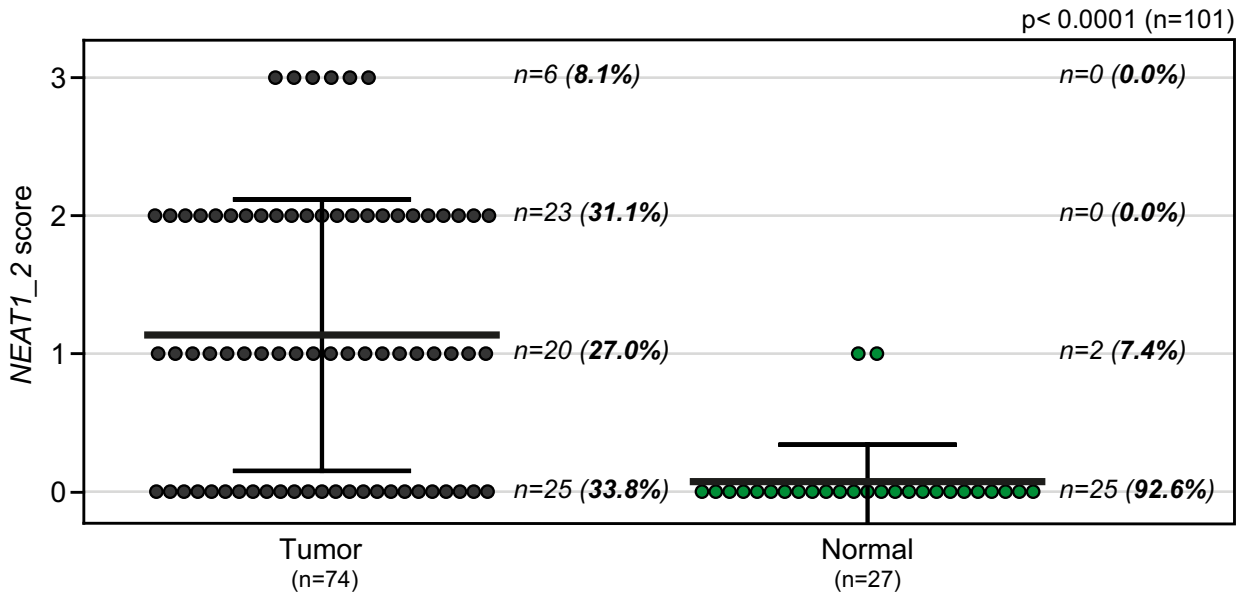

C
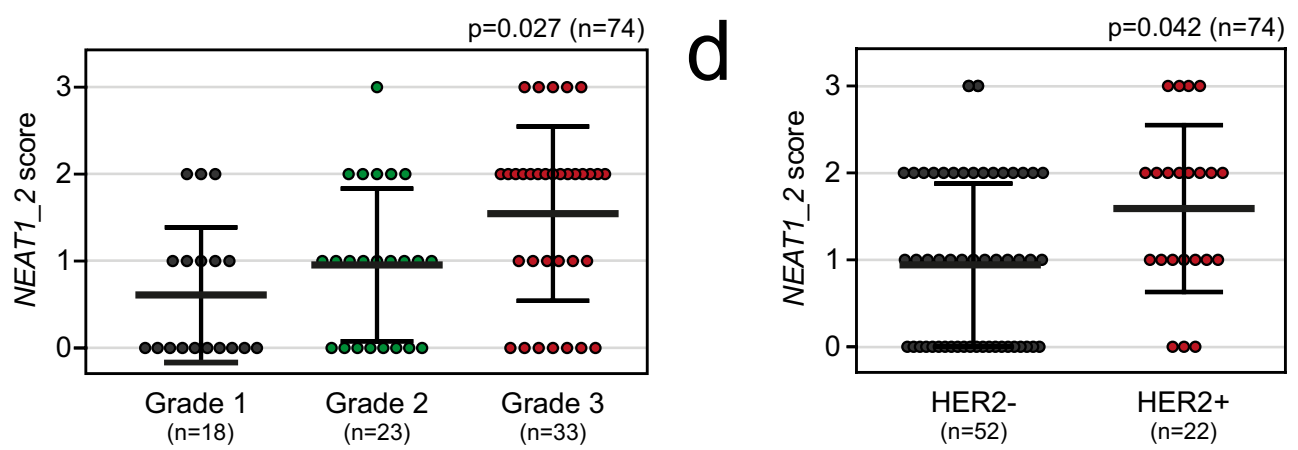

Figure 1. NEAT1_2 expression and paraspeckle formation correlate with tumor grade and HER2 expression in breast cancer. (a) RNA-FISH analyses of NEAT1_2 in formalin-fixed paraffin-embedded needle biopsies from breast cancer and benign samples. NEAT1_2 expression is scored from " 0 " to " 3 " based on punctuated nuclear NEAT1_2 signals according to the indicated criteria. For tumor samples, only tumor cells were included in the scoring, while for normal samples, only epithelial cells were included. (b) NEAT1_2 is more highly expressed in tumor versus normal tissue. (c) NEAT1_2 expression associates with tumor grade. (d) NEAT1_2 expression associates with HER2 expression. Data are presented as mean (thick black line) \pm standard deviation (thin black lines). Circles represent single patient scores. Statistical significance was calculated using the Mann Whitney test (b) or Chi square test (c) and (d). Data were considered statistically significant when $\mathrm{p} \leq 0.05$. 


\begin{tabular}{|c|c|c|c|c|c|c|c|}
\hline \multirow[b]{2}{*}{ Variable, $\mathbf{n}(\%)$} & & \multicolumn{4}{|c|}{ NEAT1_2 expression } & \multirow[b]{2}{*}{$p$} & \multirow[b]{2}{*}{ Total $(n=74)$} \\
\hline & & $0(n=25)$ & $1(n=20)$ & $2(n=23)$ & $3(n=6)$ & & \\
\hline \multirow[t]{2}{*}{ Age at diagnosis } & $<55$ & $10(34.5)$ & $8(27.6)$ & $8(27.6)$ & $3(10.3)$ & 0.920 & $29(39.2)$ \\
\hline & $\geq 55$ & $15(33.3)$ & $12(26.7)$ & $15(33.3)$ & $3(6.7)$ & & $45(60.8)$ \\
\hline \multirow[t]{3}{*}{ Histologic grade } & 1 & $10(55.6)$ & $5(27.8)$ & $3(16.7)$ & $0(0.0)$ & $0.027 *$ & $18(24.3)$ \\
\hline & 2 & $8(34.8)$ & $9(39.1)$ & $5(21.7)$ & $1(4.3)$ & & $23(31.1)$ \\
\hline & 3 & $7(22.2)$ & $6(18.2)$ & $15(45.5)$ & $5(15.2)$ & & $33(44.6)$ \\
\hline \multirow[t]{3}{*}{ Tumor type } & NST & $20(29.9)$ & $20(29.9)$ & $22(32.8)$ & $5(7.5)$ & 0.156 & $67(90.5)$ \\
\hline & ILC & $3(100.0)$ & $0(0.0)$ & $0(0.0)$ & $0(0.0)$ & & $3(4.1)$ \\
\hline & Other invasive carcinoma ${ }^{a}$ & $2(50.0)$ & $0(0.0)$ & $1(25.0)$ & $1(25.0)$ & & $4(5.4)$ \\
\hline \multirow[t]{2}{*}{ Tumor diameter $^{\mathbf{b}}$} & $<20 \mathrm{~mm}$ & $14(37.8)$ & $12(32.4)$ & $7(18.9)$ & $4(10.8)$ & 0.213 & $37(53.6)$ \\
\hline & $\geq 20 \mathrm{~mm}$ & $11(34.4)$ & $6(18.8)$ & $13(40.6)$ & $2(6.3)$ & & $32(46.4)$ \\
\hline \multirow[t]{2}{*}{ Lymph node metastasis $^{\mathbf{b}}$} & Negative & $17(35.5)$ & $14(29.2)$ & $13(27.1)$ & $4(8.3)$ & 0.990 & $48(67.6)$ \\
\hline & Positive & $8(34.8)$ & $6(26.1)$ & $7(30.4)$ & $2(8.7)$ & & $23(32.4)$ \\
\hline \multirow[t]{2}{*}{ ER } & Negative $(<1 \%)$ & $4(16.7)$ & $7(29.2)$ & $11(45.8)$ & $2(8.3)$ & 0.131 & $24(32.4)$ \\
\hline & Positive $(\geq 1 \%)$ & $21(42.0)$ & $13(26.0)$ & $12(24.0)$ & $4(8.0)$ & & $50(67.6)$ \\
\hline \multirow[t]{2}{*}{ PgR } & Negative $(<1 \%)$ & $6(20.7)$ & $8(27.6)$ & $12(41.4)$ & $3(10.3)$ & 0.226 & $29(39.2)$ \\
\hline & Positive $(\geq 1 \%)$ & $19(42.2)$ & $12(26.7)$ & $11(24.4)$ & $3(6.7)$ & & $45(60.8)$ \\
\hline \multirow[t]{2}{*}{ HER2 } & Negative $(0,1+, 2+/$ no ISH amp $)$ & $22(42.3)$ & $13(25.0)$ & $15(28.8)$ & $2(3.8)$ & $0.042 *$ & $52(70.3)$ \\
\hline & Positive (2+/ISH amp, $3+)$ & $3(13.6)$ & $7(31.8)$ & $8(36.4)$ & $4(18.2)$ & & $22(29.7)$ \\
\hline
\end{tabular}

Table 1. Clinicopathological variables and NEAT1_2 expression in breast cancer screening cohort $(\mathrm{n}=74)$. The Chi square test $\left(\chi^{2}\right.$-value) was used to calculate $p$-values. ${ }^{a}$ Tubulolobular carcinoma $(n=1)$, metaplastic squamous cell carcinoma $(n=1)$, mucinous carcinoma $(n=1)$, apocrine carcinoma $(n=1)$. ${ }^{\text {Patient }}(\mathrm{s})$ data missing. "P-value significant. Invasive carcinoma of no special type (NST), invasive lobular carcinoma (ILC), in situ hybridization (ISH), amplification (amp).

formed clusters. We therefore measured the average intensities of NEAT_2 signals per cell in all cell lines (Fig. 2e). Interestingly, HER2-positive BT474 and HCC1569 clearly expressed the highest levels of NEAT1_2. Moreover, NEAT1_2 expression levels in HER2-positive SK-BR-3 cells were only exceeded by those in MCF7 cells. This was confirmed by RT-qPCR analyses using primers specifically amplifying the NEAT1_2 isoform (Fig. 2f). Generally, results obtained by imaging and RT-qPCR were concordant, only showing deviations for the BT549 cell line. We conclude that high NEAT1_2 expression is associated with HER2-positive breast cancer and with high-grade disease. Moreover, the presence of NEAT1_2 and paraspeckles are highly specific for cancer cells, and not present or very lowly expressed in surrounding normal cells or non-cancerous breast epithelial cells.

NEAT1_2 expression is associated with the HER2-enriched and luminal B breast cancer subtypes. We demonstrated above that NEAT1_2 expression associates with HER2-positive breast cancer. HER2 overexpressing cancers are in most cases classified as HER2-enriched or luminal B using the PAM50 gene expression signature identifier. To assess the association between NEAT1_2 expression and intrinsic breast cancer subtypes, we analyzed microarray gene expression data derived from the Oslo2 cohort described above, and two publicly available breast cancer patient cohorts, METABRIC ${ }^{28}$ and The Cancer Genome Atlas (TCGA) ${ }^{45}$. Patients were subtyped using the PAM50 algorithm ${ }^{31}$, and only data generated from probes solely recognizing the NEAT1_2 isoform were considered. In all three cohorts, NEAT1_2 was most highly expressed in breast cancers classified as HER2-enriched and luminal B, but with different intrinsic distributions (HER2-enriched $>$ luminal B in Oslo2 and METABRIC; luminal B > HER2-enriched in TCGA) (Fig. 3a-c). Luminal A breast cancers had the lowest expression of NEAT1_2 in all three cohorts. Taken together, these results are in accordance with the observed correlation between NEAT1_2 expression and HER2-status.

NEAT1_1 expression is highest in luminal A and luminal B breast cancers. Previous reports have demonstrated that the NEAT1 gene is transcriptionally activated by ER $\alpha$ in both prostate and breast cancer, and the transcript participates in a gene repressor complex that induces epithelial-mesenchymal transition (EMT) in a mouse model of ER-positive breast cancer ${ }^{25,36}$. Here, we have found that the expression of the long NEAT1_2 isoform is lower in ER-positive compared to ER-negative tumors in the Oslo2 breast cancer cohort (Supplementary Fig. 1). This potential discrepancy made us analyze the expression of total NEAT1 using microarray data derived from probes binding to both NEAT1_1 and NEAT1_2 from the TCGA cohort. Interestingly, total NEAT1 expression showed a different distribution among the PAM50 subtypes compared to NEAT1_2, being most highly expressed in luminal A, luminal B, and normal-like cancers (Fig. 4). NEAT1_1 is, as opposed to NEAT1_2, a polyadenylated transcript. To more specifically investigate the expression of NEAT1_1 in breast cancer, we analyzed polyA-selected RNA-sequencing data from the TCGA breast cancer cohort. We only extracted data from samples that hardly displayed any mapping of fragments to the unique NEAT1_2 region ( $<1.0$ FPKM (Fragments Per Kilobase Million)) (Fig. 5a). Patients were then subtyped according to the PAM50 classifier, and ER and HER2 status were extracted. NEAT1_1 showed a similar distribution among the PAM50 subtypes as total NEAT1, being highest in luminal A and luminal B breast cancers (Fig. 5b). In line with this, NEAT1_1 expression 
a

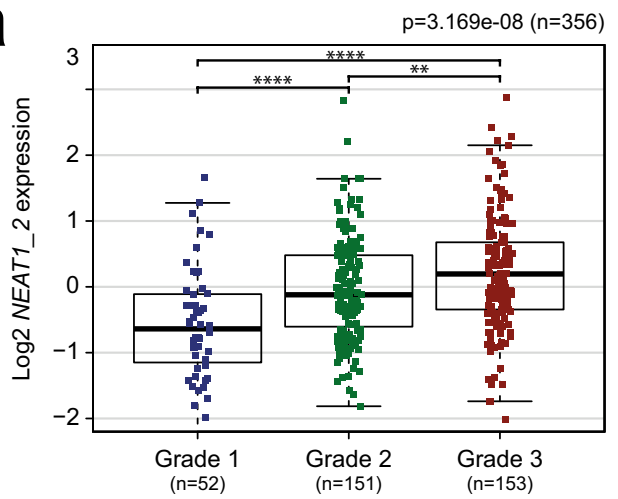

C

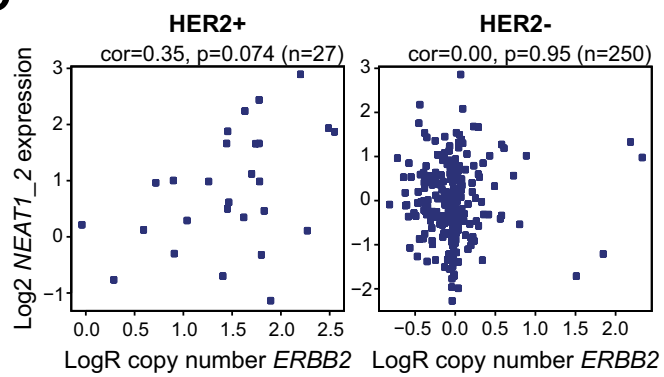

b

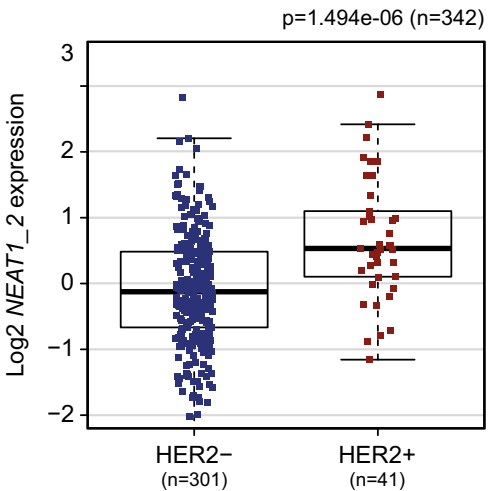

d

ERBB2 mRNA

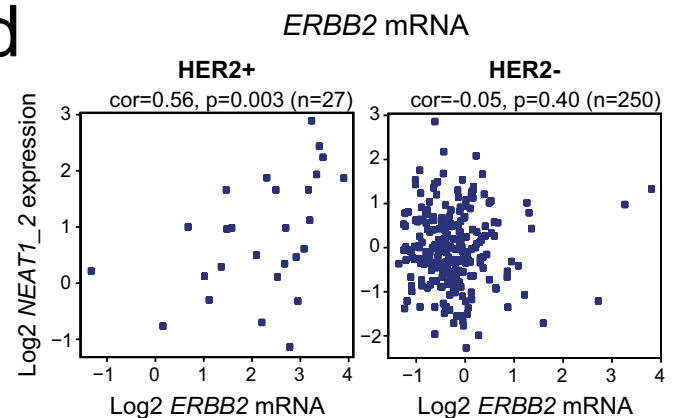

e

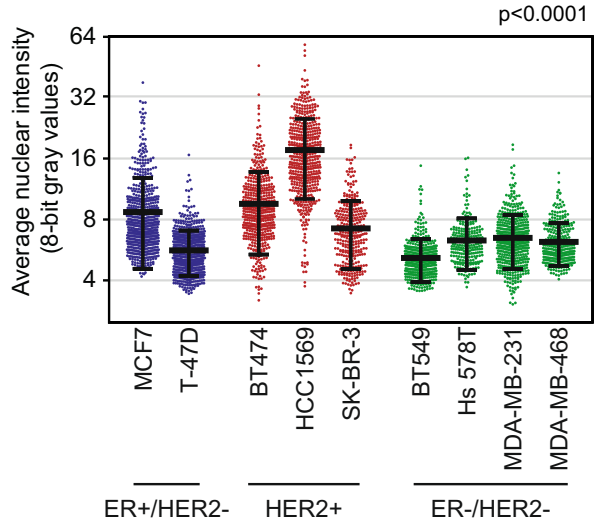

$f$

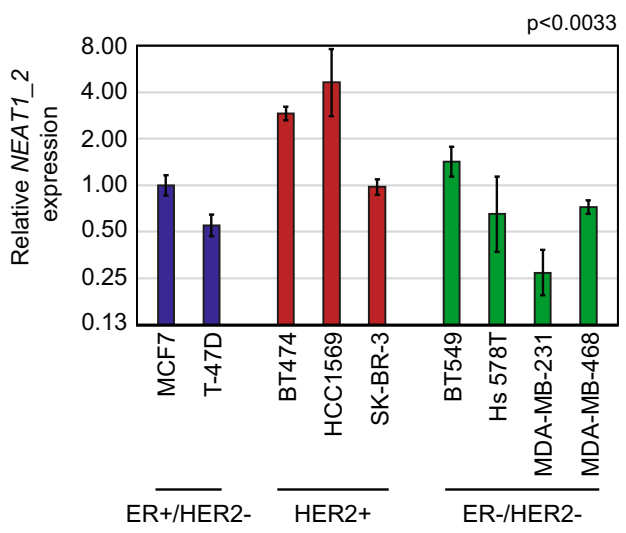

Figure 2. The association between NEAT1_2 expression and HER2 status is verified in an independent breast cancer cohort and in breast cancer cell lines. (a) NEAT1_2 expression was analyzed in microarray gene expression profiling data from patients in the Oslo2 cohort and correlated to tumor grade. The KruskalWallis test was used to calculate whether any groups are significantly different from each other and Wilcoxon Rank-Sum test was used in post-testing for significant differences between pairs of groups. $(* * * \mathrm{p} \leq 0.0001$; $* * \mathrm{p} \leq 0.01$ ). (b) NEAT1_2 expression levels were correlated to HER2 status. The Wilcoxon Rank-Sum test was used to test for significant differences between the groups. (c) and (d) NEAT1_2 expression positively correlates with ERBB2 copy number (c) and ERBB2 mRNA expression (d) in HER2-positive, but not in HER2negative, patients. Correlation was calculated using Spearman's rank correlation. (e) Breast cancer cell lines were subjected to NEAT1_2 RNA-FISH and NEAT1_2-specific signal intensity per nucleus in at least 250 cells was quantitated. Data are given as mean (thick black line) \pm standard deviation (thin black lines). Circles represent single cell intensities. The Kruskal-Wallis test was used to calculate whether any groups are significantly different from each other. (f) RNA was isolated from breast cancer cell lines and the expression of NEAT1_2 was determined by RT-qPCR. The geometric means of B2M, GAPDH, and RPLPO were used for normalization. The mean value \pm standard deviation of three biological independent experiments is presented as fold change relative to MCF7 NEAT1_2 expression. Statistical significance was calculated using the Kruskal-Wallis test. Data were considered statistically significant when $\mathrm{p} \leq 0.05$.

clearly associated with ER-positive tumors (Wilcoxon Rank-Sum test $\mathrm{p}=9.2 \mathrm{e}-37$; Fig. $5 \mathrm{c}$ ). Finally, we found no association between NEAT1_1 and HER2 expression (Wilcoxon Rank-Sum test $\mathrm{p}=0.398$; Fig. 5d). To conclude, our data clearly indicate that the relative expression of NEAT1_1 versus NEAT1_2 varies among different breast cancer subclasses. 



Figure 3. NEAT1_2 is most highly expressed in HER2-enriched and luminal B intrinsic breast cancer subtypes in three independent cohorts. (a-c) NEAT1_2 expression in PAM50 intrinsic breast cancer subtypes from patients of the Oslo2 (a), METABRIC (b), and TCGA (c) breast cancer cohorts. Subtypes were determined using the PAM50 algorithm. The Kruskal-Wallis test was used to calculate whether any groups are significantly different from each other and Wilcoxon Rank-Sum test was used in post-testing for significant differences between pairs of groups. $(* * * * \mathrm{p} \leq 0.0001 ; * * \mathrm{p} \leq 0.001 ; * * \mathrm{p} \leq 0.01 ; * \mathrm{p} \leq 0.05 ; \mathrm{ns}, \mathrm{p}>0.05)$.

NEAT1_2 expression is upregulated in human breast tissue during lactation. We have demonstrated that NEAT1_2 is rarely expressed in normal human breast tissue. NEAT1 female knock-out mice display compromised mammary gland development during puberty and pregnancy, and fail to lactate due to impaired proliferation of luminal alveolar cells $\mathrm{s}^{22}$. This suggests that NEAT1 has an important function in mammary gland development, and during pregnancy and lactation. In order to investigate if NEAT1_2 is expressed during lactation in humans, we analyzed eight needle biopsies taken from females with lactation-related benign changes in the mammary gland. Importantly, $75 \%(\mathrm{n}=6)$ of the lactating breast tissue samples were positive for NEAT1_2 using the same scoring scheme as above (Figs. 1a and 6a). Of note, we also had access to one sample from a pregnant woman, which was scored as NEAT1_2 positive (score 2). In both the lactating tissue and the breast tissue from the pregnant female, the expression of NEAT1_2 was restricted to the luminal breast epithelial cells (Fig. 6b). 

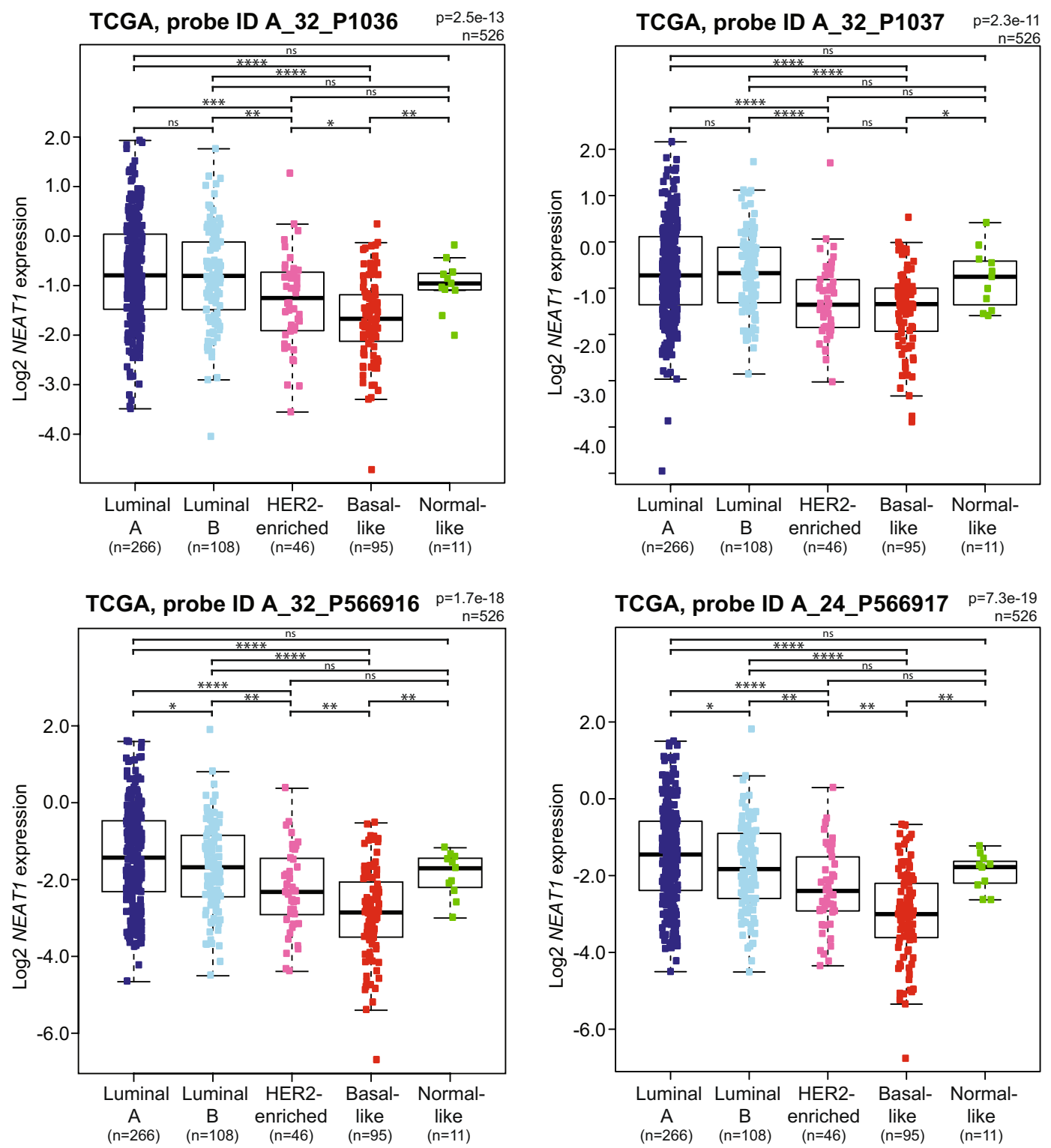

Figure 4. Total NEAT1 is most highly expressed in the luminal subtypes in the TCGA breast cancer cohort. Expression of total NEAT1 in PAM50 intrinsic breast cancer subtypes was determined using data generated from four independent microarray probes in the TCGA cohort. The Kruskal-Wallis test was used to calculate whether any groups are significantly different from each other and Wilcoxon Rank-Sum test was used in posttesting for significant differences between pairs of groups. $(* * * * p \leq 0.0001 ; * * * p \leq 0.001 ; * * p \leq 0.01 ; * p \leq 0.05$; ns, $\mathrm{p}>0.05)$.

\section{Discussion}

The lncRNA NEAT1 locus is conserved in mammalian species and encodes two overlapping transcripts, NEAT1_1 and NEAT1_2, of which the latter is essential for the assembly of paraspeckles ${ }^{1}$. Early analyses in mice indicated that whereas NEAT1_1 is ubiquitously expressed, the expression pattern of NEAT1_2, and thus the presence of paraspeckles, is more restricted ${ }^{5}$. Emerging evidence now suggests that NEAT1_2 and paraspeckles play critical roles in orchestrating specific gene expression upon cellular stress and at specific developmental stages ${ }^{9-18}$. Importantly, it was recently shown that the expression of NEAT1_2, but not total NEAT1, was associated with aggressive cancers ${ }^{15}$. Here, we have specifically analyzed the expression of NEAT1_2 in breast cancer. By performing RNA-FISH on 74 breast cancer needle biopsies, we found that NEAT1_2 expression and paraspeckle formation associated with HER2-positive cancers. We verified this by inspecting microarray data generated by a NEAT1_2-specific probe from a cohort of 381 patients. Moreover, we found that NEAT1_2 is highly expressed in HER2-positive compared to HER2-negative breast cancer cell lines. Finally, in three different breast cancer cohorts, NEAT1_2 expression associated with HER2-enriched and luminal B PAM50 intrinsic subtypes.

Around 15-20\% of all breast cancers overexpress the HER2 receptor, many of them due to the amplification of the ERBB2 gene on chromosome 17 , and HER2-driven cancers are generally aggressive ${ }^{46,47}$. The HER2 receptor is 

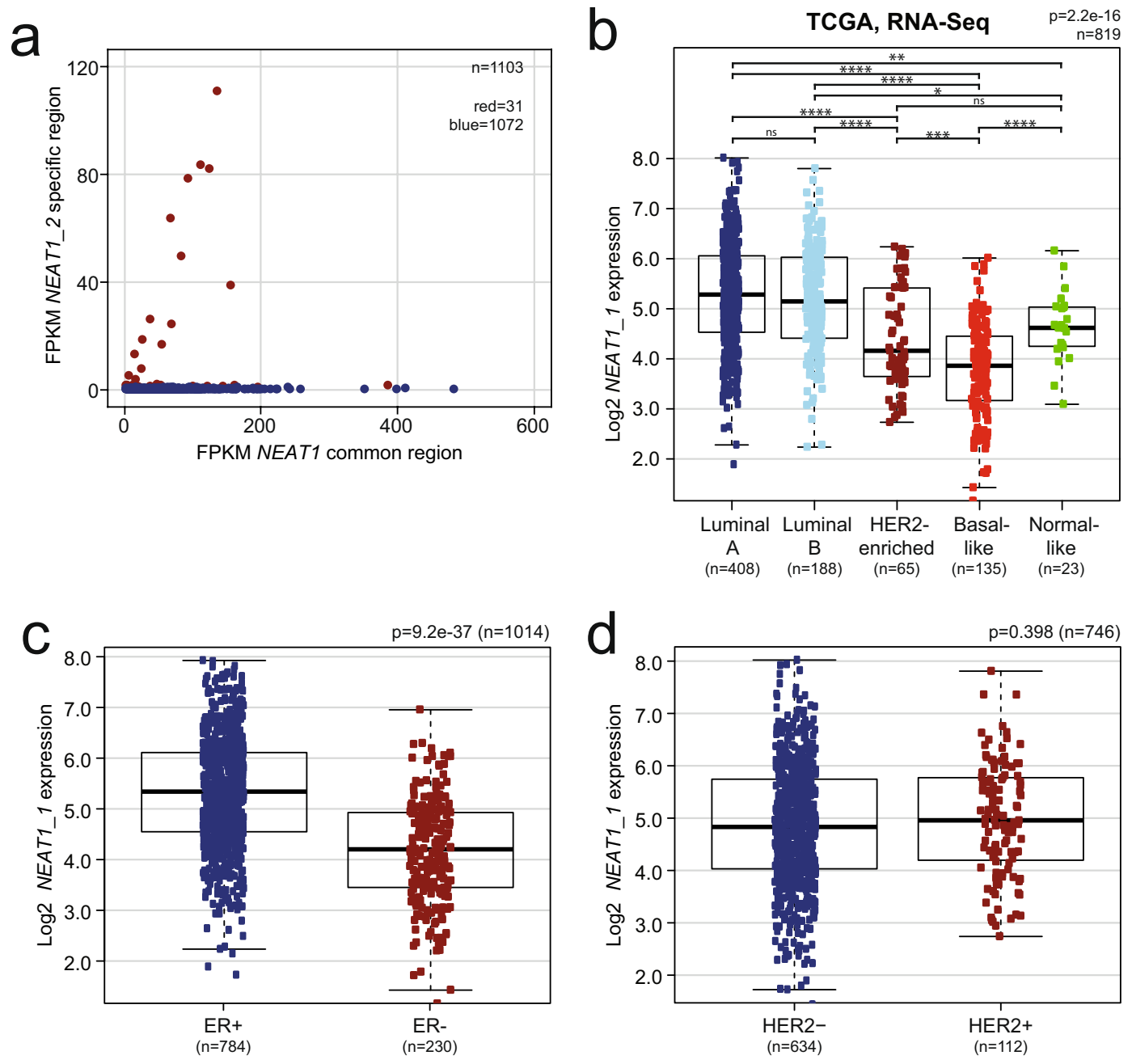

Figure 5. NEAT1_1 is most highly expressed in the luminal breast cancer subtypes in the TCGA RNA-Seq cohort. (a) NEAT1 common region FPKM (Fragments Per Kilobase Million) is plotted against NEAT1_2 specific-region FPKM for each patient. Each dot represents one patient. Samples with more than 1 FPKM for the NEAT1_2-specific region (red dots) were excluded from further analysis. (b) NEAT1_1 is most highly expressed in luminal A and luminal B tumors. The Kruskal-Wallis test was used to calculate whether any groups are significantly different from each other and Wilcoxon Rank-Sum test was used in post-testing for significant differences between pairs of groups. (c) NEAT1_1 expression associates with ER expression status. (d) NEAT1_1 expression does not associate with HER2 status. Statistical significance was calculated in (c) and (d) using Wilcoxon Rank-Sum test. Data were considered statistically significant when $\mathrm{p} \leq 0.05$. (****p $\leq 0.0001$; $* * * \mathrm{p} \leq 0.001 ; * * \mathrm{p} \leq 0.01 ; * \mathrm{p} \leq 0.05 ; \mathrm{ns}, \mathrm{p}>0.05)$.

an orphan member of the epidermal growth factor receptor family that upon overexpression forms homodimers or heterodimers with either EGFR, HER3, or HER4, which elicit signaling pathways, including the MEK-ERK and PI3-kinase-Akt pathways, that drive tumorigenesis ${ }^{46,47}$. NEAT1 expression is generally regulated at the transcriptional level, and it is reasonable to assume that HER2-signaling leads to the activation of the NEAT1 promoter. Indeed, NEAT 1 transcription is activated by a series of stress-induced transcription factors including HIF $2 \alpha$, HSF1, and NF- $\kappa B$, which have been shown to be constitutively upregulated or activated in HER2 overexpressing cells $^{48-51}$. Importantly, NEAT1 is also a p53 target gene, and oncogenic stress has been shown to upregulate NEAT1 expression in a p53-dependent manner ${ }^{15,52,53}$. This might account for the relatively high expression of NEAT1_2 observed in the wild-type p53 cell line MCF7 (Fig. 2e,f). Recently, it was suggested that high NEAT1 expression is associated with good prognosis in p53 wild-type breast cancers ${ }^{54}$. However, p53 is frequently mutated in HER2-positive cancers ${ }^{45}$, and HCC1569 cells, which express the largest amount of NEAT1_2 among the cell lines included in this study, carry a p53 nonsense mutation ${ }^{55}$. The relationship between p53 mutational status, HER2, and NEAT1 expression in breast cancer should be a subject of future research.

As NEAT1_1 and NEAT1_2 are transcribed from the same promoter, it is logical to hypothesize that the expression pattern of NEAT1_1 mirrors that of NEAT1_2. Importantly, by analyzing microarray data derived from probes binding to both NEAT1 isoforms and polyA-enriched RNA-sequencing data, we found that NEAT1_1 expression showed a different distribution among the PAM50 subtypes compared to NEAT1_2. 


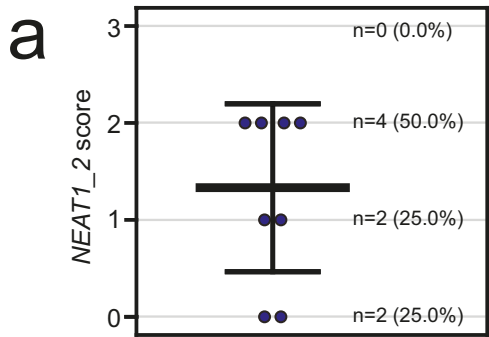

Lactating breast tissue
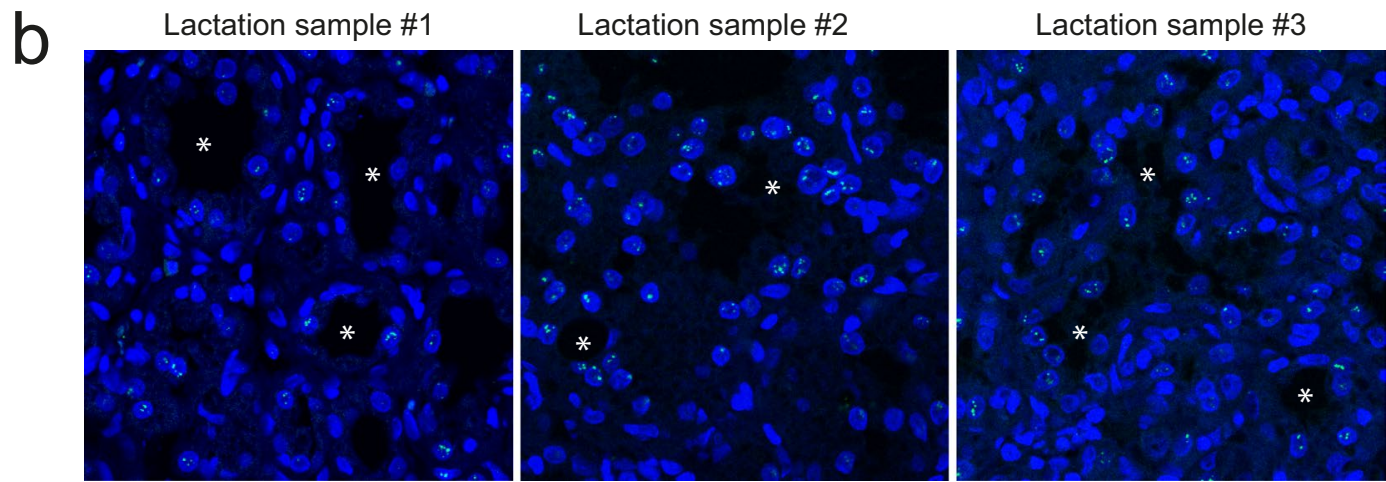

Figure 6. NEAT1_2 is expressed in lactating breast tissue. (a) RNA-FISH analyses of NEAT1_2 in breast tissue from lactating females $(\mathrm{n}=8)$. NEAT1_2 expression is scored from " 0 " to " 3 " based on punctuated nuclear NEAT1_2 signals according to the indicated criteria in Fig. 1a. Data are shown as mean (thick black line) \pm standard deviation (thin black lines). Circles represent single patient scores. (b) RNA-FISH images from three lactating females. NEAT1_2 is visualized in green, and DAPI (blue) was used to stain the nuclei. White asterisks visualize some of the lumens within the mammary glands.

Whereas NEAT1_2 is most highly expressed in HER2-enriched and luminal B cancers, NEAT1_1 expression is highest in ER-positive luminal A and luminal B cancers. Thus, our analyses strongly suggest that the relative levels of NEAT1_1 versus NEAT1_2 vary in different breast cancer subtypes. Previous reports have shown that NEAT1 is transcriptionally activated by ER $\alpha$ in both prostate and breast cancer cell lines ${ }^{25,36}$. Recently, Li et al. found that NEAT1 participates in a transcriptional repressor complex with FOXN3 and SIN3A in ER-positive breast cancer cells $^{36}$. The complex induces EMT in vitro by downregulating GATA3 expression and promotes metastasis in mouse models of ER-positive breast cancer. The FOXN3-NEAT1-SIN3A complex also binds to and represses the promoter of the ESR1 gene indicating the presence of a negative feed-back regulatory mechanism. Importantly, the authors suggest that the FOXN3-NEAT1-SIN3A complex functions independently of paraspeckles and that it is the NEAT1_1 isoform that participates in this complex. In line with this, Chakravarty et al. demonstrated that NEAT1_1, but not NEAT1_2, binds directly to histone $\mathrm{H} 3$ and recruits ER $\alpha$ to the PSMA promoter in prostate cancer cell lines ${ }^{25}$. We hypothesize that in ER-positive cancers, NEAT1_1 contributes to the tumorigenic phenotype by directly participating in transcriptional regulation at the chromatin level. This mechanism might be less important in HER2-positive cancers where increased NEAT1_2 levels and paraspeckle formation are required for their adaptation to malignancy-associated stress and survival. A recent study of pan-cancer tissue microarrays, indeed showed that $65 \%$ of human carcinomas displayed increased number of paraspeckles compared to non-malignant tissue ${ }^{15}$. More importantly, the same authors reported that the expression of NEAT1_2, but not total NEAT1, predicted progression-free survival of ovarian cancer treated with platinum-based chemotherapy. NEAT1_2 is produced when the polyadenylation signal required for the formation of NEAT1_1, is suppressed by a hnRNPK-dependent mechanism ${ }^{7,56}$. Moreover, key paraspeckle-associated proteins including NONO and SFPQ bind to and stabilize NEAT1_2 $2^{6}$. Further experiments should be undertaken to determine their expression and subcellular localization in HER2-positive breast cancers, as well as in other cancers.

We have found that NEAT1_2 is not expressed in normal tissue surrounding breast cancer cells at levels that can be detected by RNA-FISH. Furthermore, only 7.4\% of benign breast tissue samples were NEAT1_2 positive. Murine Neat 1 is critical for normal development of the mammary gland, and Neat1_2 and paraspeckles were detected in $30-50 \%$ of K8/K18-positive luminal cells in adult mice ${ }^{22}$. The number of Neat1_2 positive cells increased upon pregnancy and lactation. To further inspect NEAT1 expression pattern in human mammary gland development, we performed RNA-FISH on 8 benign breast tissue samples taken from lactating women. We detected NEAT1_2 and paraspeckles in 6 samples (75\%). Our data strongly supports the observations done in mice and suggests that NEAT1_2 and paraspeckle formation are upregulated during lactation also in humans. However, it remains to be determined at which stage in pregnancy NEAT1_2 is upregulated. As Neat1 knockout mice display compromised proliferation of luminal alveolar epithelial cells during pregnancy ${ }^{22}$, it is reasonable to hypothesize that elevated NEAT1_2 levels are required for pregnancy-induced expansion of the epithelial 
compartment in humans as well. Alternatively, NEAT1_2 might be upregulated during the differentiation of luminal alveolar cells into milk-secreting cells. The epithelium of the adult mammary gland is interspersed with mammary stem cells and progenitor cells ${ }^{57}$. In the future, experiments should be undertaken to determine the expression status of NEAT1 in these cells, as this would shed light on the function of NEAT1 in both postnatal mammary gland development and breast cancer.

We provide evidence that NEAT1_2 expression associates with HER2-positive cancers and suggest that the relative expression of NEAT1_1 versus NEAT1_2 varies in breast cancer subtypes. The overlapping nature of the NEAT1_1 and NEAT1_2 hampers isoform-specific analyses and might affect the interpretation of expression data. NEAT1_2 is not polyadenylated, which needs to be taken into account when analyzing polyA-enriched RNA-sequencing data. It should also be noted that RNA stability is a technical challenge when analyzing NEAT1_2 expression in formalin fixed paraffin embedded patient samples by RNA-FISH. Nevertheless, both NEAT1_1 and NEAT1_2 are likely to contribute to breast cancer tumorigenesis, but through different mechanisms. The highly tumor-specific expression of NEAT1_2 in breast cancer, makes it a promising target for future therapeutic intervention.

\section{Methods}

Cell culture. $\quad$ BT474 (ATCC ${ }^{\circledR}$ HTB-20 ${ }^{\mathrm{TM}}$ ), BT549 $\left(\right.$ ATCC $^{\circledR} \mathrm{HTB}^{-122^{\mathrm{TM}}}$ ), HCC1569 $\left(\right.$ ATCC $^{\circledR}$ CRL-2330 ${ }^{\mathrm{TM}}$ ), Hs

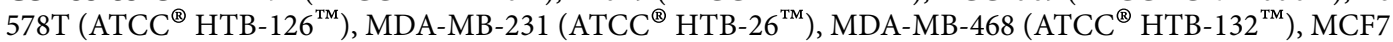
$\left(\right.$ ATCC $^{\circledR}$ HTB-22 $^{\mathrm{TM}}$ ), SK-BR-3 (ATCC ${ }^{\circledR} \mathrm{HTB}-30^{\mathrm{TM}}$ ), and T-47D $\left(\right.$ ATCC $^{\circledR}$ HTB-133 ${ }^{\mathrm{TM}}$ ) cells were all purchased from the American Type Culture Collection (ATCC). BT474, BT549, HCC1569, MDA-MB-231, MDA-MB-468, SK-BR-3, and T-47D cells were cultured in RPMI 1640 (Sigma-Aldrich) supplemented with 10\% Fetal bovine serum (FBS) (Biochrom) and 1\% penicillin-streptomycin (Sigma-Aldrich). BT549 cells were grown in the presence of $1.0 \mu \mathrm{g} / \mathrm{ml}$ insulin (Sigma-Aldrich) and T-47D cells were grown in the presence of $6.0 \mu \mathrm{g} / \mathrm{ml}$ insulin. Hs 578 T cells were cultured in Dulbecco's Modified Eagle's Medium (DMEM; Sigma-Aldrich) supplemented with $10 \%$ FBS, $1 \%$ penicillin-streptomycin, and $10.0 \mu \mathrm{g} / \mathrm{ml}$ insulin. MCF7 cells were cultured in Minimum Essential Medium Eagle (MEM; Sigma-Aldrich) supplemented with 10\% FBS, $1 \%$ penicillin-streptomycin, and $10.0 \mu \mathrm{g} / \mathrm{ml}$ insulin. All cell lines were incubated in a $5 \% \mathrm{CO} 2$ humidified incubator at $37^{\circ} \mathrm{C}$.

RNA isolation, cDNA synthesis, and RT-qPCR. Cells were lysed in $300 \mu$ l Tri Reagent (Zymo Research) and heated for $10 \mathrm{~min}$ at $55^{\circ} \mathrm{C}$ in order to prevent NEAT1 from being trapped in the protein phase during isolation $^{44}$. Total RNA was isolated with Direct-zol RNA MiniPrep (Zymo Research) according to the manufacturer's recommendation. RNA concentration was measured by NanoDrop 2000 (Thermo Fisher Scientific). cDNA synthesis of total RNA was performed with SuperScript ${ }^{\mathrm{TM}}$ IV Reverse Transcriptase (ThermoFisher Scientific). $2.5 \mu \mathrm{M}$ of random hexamer primer (ThermoFisher Scientific) and approximately $400 \mathrm{ng}$ of template were used for the reaction. Total RNA was denaturated at $65^{\circ} \mathrm{C}$ for $5 \mathrm{~min}$, and CDNA was synthesized at $50^{\circ} \mathrm{C}$ for $10 \mathrm{~min}$.

For RT-qPCR, cDNA was mixed with FastStart Essential DNA Green Master (Roche Life Science) and $0.25 \mu \mathrm{M}$ forward and reverse primer. All primer sequences are provided in Supplementary Table 1. The LightCycler ${ }^{\circledR} 96$ was used for quantification, and the $\triangle \triangle \mathrm{Cq}$-method was used to calculate fold change using the geometric mean of $G A P D H, B 2 M$, and RPLPO as internal reference.

RNA-FISH of cells and FFPE tissue. $\quad$ Stellaris ${ }^{\circledR}$ NEAT1 RNA FISH probes recognizing the NEAT1_2 isoform (SMF-2037-1 conjugated with Quasar ${ }^{\circledR}$ 670) was purchased from LGC Biosearch Technologies. Preparation of cells and FFPE sections, hybridization, and mounting were performed according to the Stellaris ${ }^{\circledR}$ RNA FISH Probes manuals. In brief, cells were seeded onto circular coverslips in 12 -well dishes and allowed to attach for 2-3 days. The cells were fixed with $4 \%$ formaldehyde and permeabilized with $70 \% \mathrm{EtOH}$. Hybridization was done at $37^{\circ} \mathrm{C}$ in a humidifying chamber for at least 4 hours. FFPE tissue sections were cut fresh and placed at $60^{\circ} \mathrm{C}$ for $45 \mathrm{~min}$ before being deparaffinized with xylene. Here, hybridization was performed overnight. Vectashield ${ }^{\circledR}$ Mounting Medium containing DAPI was used for mounting of both cells and FFPE sections. Images were generated using a Zeiss LSM780 confocal microscope. For cells, 3-dimensial Z-stack images were taken at 40x magnification (seven pictures, with $0.6 \mu \mathrm{m}$ distance between each picture). Images of FFPE sections were taken at $20 \mathrm{x}$ magnification with no Z-stacking. All images were processed using ZEN 2012 (black edition) v8.0. NEAT1_2 fluorescence was quantified from maximum intensity projections of confocal z-stacks using Fiji ${ }^{58}$ running Image ${ }^{59}$ version $1.52 \mathrm{n}$. An automatic threshold was set in the DAPI channel in order to segment individual nuclei using the wand tool. In some cases, nuclear outlines were manually traced. The average intensity in the NEAT1_2 channel was then measured for each nucleus.

Clinical samples. Archived FFPE needle biopsies were obtained from the Department of Pathology, University Hospital of North Norway (UNN) with corresponding hematoxylin and eosin (HE) slides from all patients. Samples from 74 patients diagnosed with breast cancer (2012-2018, age range 31-84 years), 27 normal samples (2013-2015, age range 18-68 years), 8 samples from lactating females (2013-2015, age range 25-42), and 1 sample from a pregnant female (2013, age 32) were included in the study. Approval for this study, including dispensation from the requirement of patient consent, was granted by the Norwegian Regional Committee for Medical and Health Research Ethics, approval number 2014/317. We confirm that all experiments were performed in accordance with relevant guidelines and regulations. Histological tumor grade was assessed by the Nottingham Grading System ${ }^{60}$. The samples were classified by pathologists as luminal A (ER+ and/or PgR+, HER2- Ki-67 < 15\%), luminal B (ER+ and/or PgR +, HER2- Ki-67 $\geq 15 \%$ or ER+ and/or PgR+, HER2+), triple negative/basal-like (ER-, PgR-, HER2-), or HER2-positive (ER-, PgR-, HER2+). The cut off values for ER and PgR were 1\%. Tumors with HER2 protein overexpression (IHC 3+) or with in situ hybridization (ISH)-detected amplified HER2 gene (IHC 2+/ISH HER2 gene amplification) were considered to be HER2 
positive. NEAT1_2 expression and clinicopathological characteristics were analyzed by the Chi square test ( $\chi^{2}$-value) using SPSS version 25 (SPSS Inc., Chicago, IL, USA). P-values $\leq 0.05$ (two-tailed) were considered statistically significant.

Gene expression analyses in breast cancer cohorts. NEAT1 gene expression was assessed in three independent breast cancer cohorts; Oslo2 ${ }^{42}, \mathrm{METABRIC}^{28}$, and $\mathrm{TCGA}^{45}$. Oslo2 is a multicentre study of breast cancer patients with primary operable breast cancers enrolled from hospitals in the Oslo (Norway) region (approved by the Norwegian Regional Committee for Medical and Health Research Ethics, approval number 2016/433 and 429-04148) ${ }^{42}$. Gene expression data (GEO accession number GSE80999) were obtained using SurePrint G3 Human GE $8 \times 60 \mathrm{~K}$ microarrays (Agilent Technologies, Santa Clara, CA, USA) and data were $\log 2$-transformed, quantile-normalized, and hospital-adjusted. Probe A_33_P3263538 covered part of the unique $3^{\prime}$ end of NEAT1_2. ERBB2 mRNA expression values were derived from mRNA probes using the median value for the two probes matching this gene symbol (A_23_P89249 and A_33_P3292596). For HER2 copy number analysis, $\log \mathrm{R}$ values (the $\log 2$-transformed value of the normalized intensity of the SNP) were extracted from raw CEL-files from Genome-Wide Human SNP Array 6.0 (Affymetrix, Santa Clara, CA, USA) using Affymetrix power tools. Segmented copy number values were generated and non-aberrant cell fraction and ploidy was calculated using the Allele-Specific Copy number Analysis of Tumors (ASCAT) package ${ }^{61}$. Segmented copy number data (adjusted for non-aberrant cell admixture and ploidy) were log2-transformed and made probe-centric based on the ERBB2 mRNA expression array probe location. The METABRIC cohort is composed of 1980 breast cancer patients collected at five different hospitals in the UK and Canada ${ }^{28}$. Gene expression was assessed using the Illumina HT-12 v3 microarray and downloaded from the European Genome-phenome Archive (EGA) data portal. The data were log2-transformed. Probe ILMN_1675354 covered part of the unique $3^{\prime}$ end of NEAT1_2. Gene expression levels for the Caucasian fraction of the TCGA cohort $(n=526)$ were assayed by Agilent $244 \mathrm{~K}$ Custom Gene Expression G4502A-07-3 ${ }^{45}$. The data were log2-transformed after normalization. The probe A_32_P206561 covered parts of the unique $3^{\prime}$ end of NEAT1_2, while probes A_32_P1036, A_32_P1037, A_24_P566917, and A_24_P566916 covered parts of the common region between NEAT1_1 and NEAT1_2. For analysis of RNA-Seq data from TCGA, bam files aligned to GRCh38 were downloaded from https://gdc.cancer.gov/. Reads mapping to the NEAT1 common region (Chr11: 65422798-65426532) and the NEAT1_2 specific region (Chr11: 6542653365445540) were counted using the featureCounts function of the Subread package specified with the -p flag (version 1.6.1 $)^{62}$. Fragments spanning the end of the NEAT1 common region and the start of the NEAT1_2 specific region were excluded. FPKM was calculated as $\left[\mathrm{RMg} * 10^{9}\right] /[\mathrm{RMt} * \mathrm{~L}]$, where $\mathrm{RMg}$ are reads mapping to each transcript, $\mathrm{L}$ is length of transcripts in bp, and RMt are total number of mapped reads. Samples with NEAT1_2 specific region FPKM $\geq 1.0$ were filtered out, leaving 1065 tumor samples for clinical analysis.

Statistical analysis. GraphPad software version 25 (SPSS Inc., Chicago, IL, USA) was used to analyze the screening cohort ( 74 breast cancer needle biopsies). Analysis of significance in expression in normal versus tumor tissue was calculated using the Mann Whitney test. For analyses of clinicopathological variables and NEAT1_2 expression the Chi square test $\left(\chi^{2}\right.$-value) were used. Data were considered statistically significant when $p \leq 0.05$.

For microarray and RNA-Seq expression analyses, statistical analyses were performed in $\mathrm{R}^{63}$. NEAT1_2, NEAT1_1, and NEAT1 expression across PAM50 intrinsic subtypes and tumor grade were compared using the non-parametric Kruskal-Wallis test. For HER2 and ER expression comparison between two groups, Wilcoxon Rank-Sum test were used. Post tests between subtypes and grade were done with Wilcoxon Rank-Sum test. Spearman's rank correlation was used for analysis of ERBB2 copy number and mRNA expression correlation with NEAT1_2 expression. Data were considered statistically significant when $\mathrm{p} \leq 0.05$.

Received: 8 September 2019; Accepted: 21 December 2019;

Published online: 28 January 2020

\section{References}

1. Hutchinson, J. N. et al. A screen for nuclear transcripts identifies two linked noncoding RNAs associated with SC35 splicing domains. BMC Genomics 8, 39, https://doi.org/10.1186/1471-2164-8-39 (2007).

2. Sasaki, Y. T., Ideue, T., Sano, M., Mituyama, T. \& Hirose, T. MENepsilon/beta noncoding RNAs are essential for structural integrity of nuclear paraspeckles. Proc. Natl Acad. Sci. USA 106, 2525-2530, https://doi.org/10.1073/pnas.0807899106 (2009).

3. Sunwoo, H. et al. MEN epsilon/beta nuclear-retained non-coding RNAs are up-regulated upon muscle differentiation and are essential components of paraspeckles. Genome Res. 19,347-359, https://doi.org/10.1101/gr.087775.108 (2009).

4. Hirose, T., Yamazaki, T. \& Nakagawa, S. Molecular anatomy of the architectural NEAT1 noncoding RNA: The domains, interactors, and biogenesis pathway required to build phase-separated nuclear paraspeckles. Wiley Interdiscip Rev RNA, e1545, https://doi. org/10.1002/wrna.1545 (2019).

5. Nakagawa, S., Naganuma, T., Shioi, G. \& Hirose, T. Paraspeckles are subpopulation-specific nuclear bodies that are not essential in mice. J. Cell Biol. 193, 31-39, https://doi.org/10.1083/jcb.201011110 (2011).

6. Yamazaki, T. et al. Functional Domains of NEAT1 Architectural lncRNA Induce Paraspeckle Assembly through Phase Separation. Mol. Cell 70, 1038-1053 e1037, https://doi.org/10.1016/j.molcel.2018.05.019 (2018).

7. Naganuma, T. et al. Alternative $3^{\prime}$-end processing of long noncoding RNA initiates construction of nuclear paraspeckles. EMBO J. 31, 4020-4034, https://doi.org/10.1038/emboj.2012.251 (2012).

8. Li, R., Harvey, A. R., Hodgetts, S. I. \& Fox, A. H. Functional dissection of NEAT1 using genome editing reveals substantial localization of the NEAT1_l isoform outside paraspeckles. RNA 23, 872-881, https://doi.org/10.1261/rna.059477.116 (2017).

9. Beeharry, Y., Goodrum, G., Imperiale, C. J. \& Pelchat, M. The Hepatitis Delta Virus accumulation requires paraspeckle components and affects NEAT1 level and PSP1 localization. Sci. Rep. 8, 6031, https://doi.org/10.1038/s41598-018-24500-1 (2018).

10. Saha, S., Murthy, S. \& Rangarajan, P. N. Identification and characterization of a virus-inducible non-coding RNA in mouse brain. J. Gen. Virol. 87, 1991-1995, https://doi.org/10.1099/vir.0.81768-0 (2006).

11. Zhang, Q., Chen, C. Y., Yedavalli, V. S. \& Jeang, K. T. NEAT1 long noncoding RNA and paraspeckle bodies modulate HIV-1 posttranscriptional expression. MBio 4, e00596-00512, https://doi.org/10.1128/mBio.00596-12 (2013). 
12. Lellahi, S. M. et al. The long noncoding RNA NEAT1 and nuclear paraspeckles are up-regulated by the transcription factor HSF1 in the heat shock response. J. Biol. Chem. 293, 18965-18976, https://doi.org/10.1074/jbc.RA118.004473 (2018).

13. Ma, H. et al. The Long Noncoding RNA NEAT1 Exerts Antihantaviral Effects by Acting as Positive Feedback for RIG-I Signaling. J Virol 91, https://doi.org/10.1128/JVI.02250-16 (2017).

14. Wang, Y. et al. Genome-wide screening of NEAT1 regulators reveals cross-regulation between paraspeckles and mitochondria. Nat. Cell Biol. 20, 1145-1158, https://doi.org/10.1038/s41556-018-0204-2 (2018).

15. Adriaens, C. et al. p53 induces formation of NEAT1 lncRNA-containing paraspeckles that modulate replication stress response and chemosensitivity. Nat. Med. 22, 861-868, https://doi.org/10.1038/nm.4135 (2016).

16. Choudhry, H. et al. Tumor hypoxia induces nuclear paraspeckle formation through HIF-2alpha dependent transcriptional activation of NEAT1 leading to cancer cell survival. Oncogene 34, 4546, https://doi.org/10.1038/onc.2014.431 (2015).

17. Hirose, T. et al. NEAT1 long noncoding RNA regulates transcription via protein sequestration within subnuclear bodies. Mol. Biol. Cell 25, 169-183, https://doi.org/10.1091/mbc.E13-09-0558 (2014).

18. Imamura, K. et al. Long noncoding RNA NEAT1-dependent SFPQ relocation from promoter region to paraspeckle mediates IL8 expression upon immune stimuli. Mol. Cell 53, 393-406, https://doi.org/10.1016/j.molcel.2014.01.009 (2014).

19. Chen, L. L. \& Carmichael, G. G. Altered nuclear retention of mRNAs containing inverted repeats in human embryonic stem cells: functional role of a nuclear noncoding RNA. Mol. Cell 35, 467-478, https://doi.org/10.1016/j.molcel.2009.06.027 (2009).

20. Prasanth, K. V. et al. Regulating gene expression through RNA nuclear retention. Cell 123, 249-263, https://doi.org/10.1016/j. cell.2005.08.033 (2005).

21. Nakagawa, S. et al. The lncRNA Neat1 is required for corpus luteum formation and the establishment of pregnancy in a subpopulation of mice. Development 141, 4618-4627, https://doi.org/10.1242/dev.110544 (2014).

22. Standaert, L. et al. The long noncoding RNA Neat1 is required for mammary gland development and lactation. RNA 20, 1844-1849, https://doi.org/10.1261/rna.047332.114 (2014).

23. Hupalowska, A. et al. CARM1 and Paraspeckles Regulate Pre-implantation Mouse Embryo Development. Cell 175, 1902-1916 e1913, https://doi.org/10.1016/j.cell.2018.11.027 (2018).

24. Hanahan, D. \& Weinberg, R. A. Hallmarks of cancer: the next generation. Cell 144, 646-674, https://doi.org/10.1016/j. cell.2011.02.013 (2011).

25. Chakravarty, D. et al. The oestrogen receptor alpha-regulated lncRNA NEAT1 is a critical modulator of prostate cancer. Nat. Commun. 5, 5383, https://doi.org/10.1038/ncomms6383 (2014).

26. Klec, C., Prinz, F. \& Pichler, M. Involvement of the long noncoding RNA NEAT1 in carcinogenesis. Mol. Oncol. 13, 46-60, https:// doi.org/10.1002/1878-0261.12404 (2019).

27. Koren, S. \& Bentires-Alj, M. Breast Tumor Heterogeneity: Source of Fitness, Hurdle for Therapy. Mol. Cell 60, 537-546, https://doi. org/10.1016/j.molcel.2015.10.031 (2015).

28. Curtis, C. et al. The genomic and transcriptomic architecture of 2,000 breast tumours reveals novel subgroups. Nature 486, 346-352, https://doi.org/10.1038/nature10983 (2012).

29. Perou, C. M. et al. Molecular portraits of human breast tumours. Nature 406, 747-752, https://doi.org/10.1038/35021093 (2000).

30. Sorlie, T. et al. Gene expression patterns of breast carcinomas distinguish tumor subclasses with clinical implications. Proc. Natl Acad. Sci. USA 98, 10869-10874, https://doi.org/10.1073/pnas.191367098 (2001).

31. Parker, J. S. et al. Supervised risk predictor of breast cancer based on intrinsic subtypes. J. Clin. Oncol. 27, 1160-1167, https://doi. org/10.1200/JCO.2008.18.1370 (2009)

32. Ke, H. et al. NEAT1 is Required for Survival of Breast Cancer Cells Through FUS and miR-548. Gene Regul. Syst. Bio 10, 11-17, https://doi.org/10.4137/GRSB.S29414 (2016).

33. Jiang, X., Zhou, Y., Sun, A. J. \& Xue, J. L. NEAT1 contributes to breast cancer progression through modulating miR-448 and ZEB1. J. Cell Physiol. 233, 8558-8566, https://doi.org/10.1002/jcp.26470 (2018).

34. Qian, K. et al. The long non-coding RNA NEAT1 interacted with miR-101 modulates breast cancer growth by targeting EZH2. Arch. Biochem. Biophys. 615, 1-9, https://doi.org/10.1016/j.abb.2016.12.011 (2017)

35. Zhao, D., Zhang, Y., Wang, N. \& Yu, N. NEAT1 negatively regulates miR-218 expression and promotes breast cancer progression. Cancer Biomark 20, 247-254, https://doi.org/10.3233/CBM-170027 (2017).

36. Li, W. et al. The FOXN3-NEAT1-SIN3A repressor complex promotes progression of hormonally responsive breast cancer. J. Clin. Invest. 127, 3421-3440, https://doi.org/10.1172/JCI94233 (2017).

37. Shin, V. Y. et al. Long non-coding RNA NEAT1 confers oncogenic role in triple-negative breast cancer through modulating chemoresistance and cancer stemness. Cell Death Dis. 10, 270, https://doi.org/10.1038/s41419-019-1513-5 (2019).

38. Rheinbay, E. et al. Recurrent and functional regulatory mutations in breast cancer. Nature 547, 55-60, https://doi.org/10.1038/ nature22992 (2017).

39. Nik-Zainal, S. et al. Landscape of somatic mutations in 560 breast cancer whole-genome sequences. Nature 534, 47-54, https://doi. org/10.1038/nature17676 (2016).

40. Adriaens, C. et al. The long noncoding RNA NEAT1_l is seemingly dispensable for normal tissue homeostasis and cancer cell growth. RNA 25, 1681-1695, https://doi.org/10.1261/rna.071456.119 (2019).

41. Isobe, M. et al. Forced isoform switching of Neat1_1 to Neat1_2 leads to the loss of Neat1_1 and the hyperformation of paraspeckles but does not affect the development and growth of mice. bioRxiv, 698068, https://doi.org/10.1101/698068 (2019).

42. Aure, M. R. et al. Integrative clustering reveals a novel split in the luminal A subtype of breast cancer with impact on outcome. Breast Cancer Res. 19, 44, https://doi.org/10.1186/s13058-017-0812-y (2017).

43. Neve, R. M. et al. A collection of breast cancer cell lines for the study of functionally distinct cancer subtypes. Cancer Cell 10, 515-527, https://doi.org/10.1016/j.ccr.2006.10.008 (2006).

44. Chujo, T. et al. Unusual semi-extractability as a hallmark of nuclear body-associated architectural noncoding RNAs. EMBO J. 36, 1447-1462, https://doi.org/10.15252/embj.201695848 (2017).

45. Cancer Genome Atlas, N. Comprehensive molecular portraits of human breast tumours. Nature 490, 61-70, https://doi.org/10.1038/ nature11412 (2012)

46. Gutierrez, C. \& Schiff, R. HER2: biology, detection, and clinical implications. Arch. Pathol. Lab. Med. 135, 55-62, https://doi. org/10.1043/2010-0454-RAR.1 (2011).

47. Moasser, M. M. The oncogene HER2: its signaling and transforming functions and its role in human cancer pathogenesis. Oncogene 26, 6469-6487, https://doi.org/10.1038/sj.onc.1210477 (2007).

48. Jarman, E. J. et al. HER2 regulates HIF-2alpha and drives an increased hypoxic response in breast cancer. Breast Cancer Res. 21, 10, https://doi.org/10.1186/s13058-019-1097-0 (2019).

49. Schulz, R. et al. HER2/ErbB2 activates HSF1 and thereby controls HSP90 clients including MIF in HER2-overexpressing breast cancer. Cell Death Dis. 5, e980, https://doi.org/10.1038/cddis.2013.508 (2014).

50. Xi, C., Hu, Y., Buckhaults, P., Moskophidis, D. \& Mivechi, N. F. Heat shock factor Hsf1 cooperates with ErbB2 (Her2/Neu) protein to promote mammary tumorigenesis and metastasis. J. Biol. Chem. 287, 35646-35657, https://doi.org/10.1074/jbc.M112.377481 (2012).

51. Cogswell, P. C., Guttridge, D. C., Funkhouser, W. K. \& Baldwin, A. S. Jr. Selective activation of NF-kappa B subunits in human breast cancer: potential roles for NF-kappa B2/p52 and for Bcl-3. Oncogene 19, 1123-1131, https://doi.org/10.1038/s.onc.1203412 (2000). 
52. Mello, S. S. et al. Neat1 is a p53-inducible lincRNA essential for transformation suppression. Genes. Dev. 31, 1095-1108, https://doi. org/10.1101/gad.284661.116 (2017).

53. Idogawa, M., Ohashi, T., Sasaki, Y., Nakase, H. \& Tokino, T. Long non-coding RNA NEAT1 is a transcriptional target of p53 and modulates p53-induced transactivation and tumor-suppressor function. Int. J. Cancer 140, 2785-2791, https://doi.org/10.1002/ ijc.30689 (2017).

54. Idogawa, M., Nakase, H., Sasaki, Y. \& Tokino, T. Prognostic Effect of Long Noncoding RNA NEAT1 Expression Depends on p53 Mutation Status in Cancer. J Oncol 2019, 4368068, https://doi.org/10.1155/2019/4368068 (2019).

55. Lacroix, M., Toillon, R. A. \& Leclercq, G. p53 and breast cancer, an update. Endocr. Relat. Cancer 13, 293-325, https://doi. org/10.1677/erc.1.01172 (2006).

56. Naganuma, T. \& Hirose, T. Paraspeckle formation during the biogenesis of long non-coding RNAs. RNA Biol. 10, 456-461, https:// doi.org/10.4161/rna.23547 (2013).

57. Fu, N., Nolan, E., Lindeman, G. J. \& Visvader, J. E. Stem Cells and the Differentiation Hierarchy in Mammary Gland Development. Physiol Rev, https://doi.org/10.1152/physrev.00040.2018 (2019).

58. Schindelin, J. et al. Fiji: an open-source platform for biological-image analysis. Nat. Methods 9, 676-682, https://doi.org/10.1038/ nmeth.2019 (2012).

59. Schneider, C. A., Rasband, W. S. \& Eliceiri, K. W. NIH Image to ImageJ: 25 years of image analysis. Nat. Methods 9, 671-675 (2012).

60. Elston, C. W. \& Ellis, I. O. Pathological prognostic factors in breast cancer. I. The value of histological grade in breast cancer: experience from a large study with long-term follow-up. Histopathology 19, 403-410 (1991).

61. Van Loo, P. et al. Allele-specific copy number analysis of tumors. Proc. Natl Acad. Sci. USA 107, 16910-16915, https://doi. org/10.1073/pnas.1009843107 (2010).

62. Liao, Y., Smyth, G. K. \& Shi, W. featureCounts: an efficient general purpose program for assigning sequence reads to genomic features. Bioinformatics 30, 923-930, https://doi.org/10.1093/bioinformatics/btt656 (2014).

63. R: A language and environment for statistical computing (R Foundation for Statistical Computing, Vienna, Austria, 2018).

\section{Acknowledgements}

This work was supported by Northern Norway Regional Health Authority (HNF1371-17). The publication charges for this article have been funded by a grant from the publication fund of UiT - The Arctic University of Norway.

\section{Author contributions}

Conceived and designed the experiments: E.K., M.P. Performed the experiments: E.K., S.M.L., S.F., A.H. Analyzed the data: E.K., M.R.A., S.N., S.F., K.B.L., M.T.G., A.H., S.S.B., E.S.M. and M.P. Contributed reagents/materials/ analysis tools: OSBREAC, A.M.B., G.M.M., T.S., E.S.M., M.P. Wrote the paper: E.K., M.P. All authors reviewed the manuscript.

\section{Competing interests}

The authors declare no competing interests.

\section{Additional information}

Supplementary information is available for this paper at https://doi.org/10.1038/s41598-020-57759-4.

Correspondence and requests for materials should be addressed to M.P.

Reprints and permissions information is available at www.nature.com/reprints.

Publisher's note Springer Nature remains neutral with regard to jurisdictional claims in published maps and institutional affiliations.

Open Access This article is licensed under a Creative Commons Attribution 4.0 International License, which permits use, sharing, adaptation, distribution and reproduction in any medium or format, as long as you give appropriate credit to the original author(s) and the source, provide a link to the Creative Commons license, and indicate if changes were made. The images or other third party material in this article are included in the article's Creative Commons license, unless indicated otherwise in a credit line to the material. If material is not included in the article's Creative Commons license and your intended use is not permitted by statutory regulation or exceeds the permitted use, you will need to obtain permission directly from the copyright holder. To view a copy of this license, visit http://creativecommons.org/licenses/by/4.0/.

(c) The Author(s) 2020 


\section{Consortia}

\section{Oslo Breast Cancer Research Consortium (OSBREAC)}

Jürgen Geisler ${ }^{7,8,9}$, Solveig Hofvind ${ }^{10,11}$, Tone F. Bathen ${ }^{12}$, Elin Borgen ${ }^{13}$, Anne-Lise BørresenDale $^{2,8}$, Olav Engebråten ${ }^{6,8,14}$, Øystein Garred ${ }^{13}$, Gry Aarum Geitvik ${ }^{2}$, Anita Langerød ${ }^{2}$, Bjørn Naume $^{8,14}$, Hege G. Russnes ${ }^{2,13}$, Ellen Schlichting ${ }^{15}$, Ole Christian Lingjærde ${ }^{16,17}$, Vessela N. Kristensen ${ }^{2,8,18}$, Helle Kristine Skjerven ${ }^{19}$, Thomas Papathomas ${ }^{20}$, Olaf-Johan HartmanJohnsen $^{21} \&$ Kristine Kleivi Sahlberg ${ }^{22}$

${ }^{7}$ Department of Oncology, Akershus University Hospital, Lørenskog, Norway. ${ }^{8}$ Institute for Clinical Medicine, Faculty of Medicine, University of Oslo, Oslo, Norway. ${ }^{9}$ Division of Medicine, Akershus University Hospital, Lørenskog, Norway. ${ }^{10}$ Cancer Registry of Norway, Oslo, Norway. ${ }^{11}$ Oslo and Akershus University College of Applied Sciences, Faculty of Health Science, Oslo, Norway. ${ }^{12}$ Department of Circulation and Medical Imaging, Norwegian University of Science and Technology (NTNU), Trondheim, Norway. ${ }^{13}$ Department of Pathology, Division of Diagnostics and Intervention, Oslo University Hospital, Oslo, Norway. ${ }^{14}$ Department of Oncology, Division of Surgery and Cancer and Transplantation Medicine, Oslo University Hospital, Oslo, Norway. ${ }^{15}$ Section for Breast- and Endocrine Surgery, Department of Cancer, Division of Surgery, Cancer and Transplantation Medicine, Oslo University Hospital, Oslo, Norway. ${ }^{16}$ Centre for Cancer Biomedicine, University of Oslo, Oslo, Norway. ${ }^{17}$ Department of Computer Science, University of Oslo, Oslo, Norway. ${ }^{18}$ Department of Clinical Molecular Biology and Laboratory Science (EpiGen), Division of Medicine, Akershus University Hospital, Lørenskog, Norway. ${ }^{19} 0$ Breast and Endocrine Surgery, Department of Breast and Endocrine Surgery, Vestre Viken Hospital Trust, Drammen, Norway. ${ }^{20}$ Department of Pathology, Vestre Viken Hospital Trust, Drammen, Norway. ${ }^{21}$ Østfold Hospital, Østfold, Norway. ${ }^{22}$ Department of Research, Vestre Viken Hospital Trust, Drammen, Norway. 